\title{
Effective thermal dynamics following a quantum quench in a spin chain
}

\author{
Davide Rossini, ${ }^{1}$ Alessandro Silva, ${ }^{2}$ Giuseppe Mussardo, ${ }^{1,2,3}$ and Giuseppe E. Santoro ${ }^{1,2}$ \\ ${ }^{1}$ International School for Advanced Studies (SISSA), Via Beirut 2-4, I-34014 Trieste, Italy \\ ${ }^{2}$ International Centre for Theoretical Physics (ICTP), I-34014 Trieste, Italy \\ ${ }^{3}$ Istituto Nazionale di Fisica Nucleare, Sezione di Trieste, Trieste, Italy
}

\begin{abstract}
We study the nonequilibrium dynamics of the Quantum Ising Model following an abrupt quench of the transverse field. We focus on the onsite autocorrelation function of the order parameter, and extract the phase coherence time $\tau_{Q}^{\varphi}$ from its asymptotic behavior. We show that the initial state determines $\tau_{Q}^{\varphi}$ only through an effective temperature set by its energy and the final Hamiltonian. Moreover, we observe that the dependence of $\tau_{Q}^{\varphi}$ on the effective temperature fairly agrees with that obtained in thermal equilibrium as a function of the equilibrium temperature.
\end{abstract}

PACS numbers: 75.40.Gb, 75.10.Pq, 73.43.Nq, 03.65.Sq

A recent series of beautiful experiments with cold atomic gases [1, 2, 3] have triggered a great deal of interest in some fundamental aspects of the non-equilibrium dynamics of correlated quantum systems. The peculiarity of the dynamics of cold atoms is its phase coherence on long time scales. This was clearly demonstrated by the cycles of collapse and revival of the order parameter observed in Ref. [2]. The interplay between phase coherence, strong interactions, and low dimensionality may result in surprising effects: an example is the lack of thermalization recently observed in quasi-one dimensional condensates [3]. The attribution of this phenomenon to the closeness of these systems to integrability spurred an intense discussion on the general relation between quantum integrability and thermalization in the long-time dynamics of strongly correlated quantum systems [4, 5, 6, 7, 8, 9, 10, 11, 12, 13, 14, 15].

The simplest nonequilibrium process to be considered in order to study the long-time dynamics of a quantum system is the quantum quench: an abrupt change in time of one of the system parameters or of its boundary conditions. Recent studies of strongly correlated models [4, 5, 6, 7, 8, 9, 10, 11, 12, 13, 14, 15] have demonstrated that the behavior of integrable and nonintegrable systems can be quite different. Thermalization can be observed, under specific circumstances, in nonintegrable systems [8, 9, 10]: asymptotic values of significant observables, such as the momentum distribution function, do not depend on the details of the initial state, but only on its energy [8]. On the other hand, for integrable systems thermalization does not oc$\operatorname{cur}[5,6,7,11,12,13,14,15]$ : a larger amount of information on the initial state seems necessary to predict the asymptotic state. It has been conjectured that this information consists of the expectation value of a set of constants of motion fixing in the Lagrange multipliers of a generalized Gibbs ensemble 7]. For a special quench in a 1D Bose-Hubbard model [11] and for integrable systems with free quasiparticles [12], the local reduced density matrix was indeed proven to asymptotically tend to such generalized ensemble. Moreover, the generalized Gibbs ensemble was shown to correctly predict the asymptotic momentum distribution functions for a variety of models and quenches [6, 7, 13, 14]. However, it should be pointed out that neglection of correlations of the occupation of different quasi-particle modes leads to incorrect predictions for the noise and higher order correlators [15].

In this Letter, instead of focusing on the asymptotics of observables, we take a different perspective, and study the dependence on the initial state $\left|\psi_{0}\right\rangle$ of the intrinsic time-scale of the dynamics after the quench. We do this by considering the Quantum Ising chain, a prototypical example of exactly solvable model with a quantum phase transition [16]. We study the autocorrelation function of the order parameter after a quench of the transverse field, extracting the phase coherence time $\tau_{Q}^{\varphi}$ from its asymptotic exponential decay. We will show that, regardless of the integrability of the model, the only information on the quench needed to predict $\tau_{Q}^{\varphi}$ is the final gap $\Delta$ and an effective temperature $T_{\text {eff }}$, determined by the energy of the initial state after the quench; moreover, the dependence of $\tau_{Q}^{\varphi}$ on $T_{\text {eff }}$ is in very good agreement with that obtained, at equilibrium, for the same quantity $\tau_{T}^{\varphi}$ as a function of the equilibrium temperature $T$.

The sharp contrast between the asymptotics of observables like the transverse magnetization, determined by the entire set of constants of motion, and the phase coherence time $\tau_{Q}^{\varphi}$, depending just on $T_{\text {eff }}$, has its deep roots in the physics of the Quantum Ising chain [16]. This model, which can be diagonalized in the continuum limit in terms of Majorana fermion quasi-particles (see, e.g. 17]), possesses two sectors of operators [18]: a local sector with respect to the quasiparticles, where the $S$-matrix is simply $S=1$ and the model is equivalent to a free theory, and a non-local sector, where $S=-1$ and the model describes an interacting theory. In this respect, the non-local sector can be used as low-energy theory of a more general class of models, not necessarily integrable, belonging to the Ising universality class (e.g., the nonintegrable $\Phi^{4}$ Landau-Ginzburg model [19]). While the transverse magnetization belongs to the local sector, im- 
plying sensitivity of its asymptotics to integrability, the order parameter belongs to the nonlocal sector, making $\tau_{Q}^{\varphi}$ representative of the Ising universality class.

We start by considering a spin-1/2 Quantum Ising chain in a transverse magnetic field $\Gamma$ with periodic boundary conditions:

$$
\mathcal{H}(\Gamma)=-J \sum_{j}\left[\sigma_{j}^{x} \sigma_{j+1}^{x}+\Gamma \sigma_{j}^{z}\right],
$$

where $\sigma_{j}^{\alpha}(\alpha=x, y, z)$ are spin operators, $J$ is the interaction strength. Hereafter, unless explicitly written, we set $J=1$. This system has a quantum critical point at $\Gamma_{c}=1$ separating two mutually dual gapped phases, a quantum paramagnetic one $(\Gamma>1)$ and a ferromagnetic one $(\Gamma<1)$, with energy gap $\Delta \equiv 2|1-\Gamma|$. At equilibrium, the presence of a critical point dramatically influences the temperature dependence of the basic time-scale characterizing the system's dynamics: the phase coherence time $\tau_{T}^{\varphi}$ [16]. The latter is usually extracted from the asymptotics of the on-site spin autocorrelation function $\rho_{T}^{x x}(t) \equiv\left\langle\sigma_{j}^{x}(t) \sigma_{j}^{x}(0)\right\rangle$, which decays to zero exponentially, [20, 21] $\rho_{T}^{x x}(t) \sim e^{-t / \tau_{T}^{\varphi}}$, at any finite temperature $T>0$, both at criticality $(\Delta=0)$, and in the off-critical region $(T \ll \Delta)$. At criticality [20], for $T \ll J$ one finds $\tau_{T}^{\varphi} \simeq \frac{8}{\pi T}$, while $\tau_{T}^{\varphi}$ is exponentially larger [21, 22] in the off-critical region with $T \ll \Delta: \tau_{T}^{\varphi} \simeq \frac{\pi}{2 T} e^{\Delta / T}$.

Consider now a quantum quench, which consists of preparing the system in the ground state corresponding to a transverse field $\Gamma_{0},\left|\psi_{0}\right\rangle=\left|\psi\left(\Gamma_{0}\right)\right\rangle$, and then abruptly quenching it, at $t=0$, to some $\Gamma \neq \Gamma_{0}$. For $t>0$, the state evolves unitarily under $\mathcal{H}(\Gamma)$, according to $\left|\psi_{t}\right\rangle=\exp [-i \mathcal{H}(\Gamma) t]\left|\psi\left(\Gamma_{0}\right)\right\rangle$. We define the zerotemperature on-site autocorrelation function describing the spin dynamics after the quench:

$$
\rho_{Q}^{x x}(t) \equiv\left\langle\psi\left(\Gamma_{0}\right)\left|e^{i \mathcal{H}(\Gamma) t} \sigma_{j}^{x} e^{-i \mathcal{H}(\Gamma) t} \sigma_{j}^{x}\right| \psi\left(\Gamma_{0}\right)\right\rangle .
$$

Before entering into details, we summarize the results obtained by analyzing the asymptotics of $\rho_{Q}^{x x}(t)$ : it always drops exponentially to zero (see Fig. 1), $\rho_{Q}^{x x}(t) \sim e^{-t / \tau_{Q}^{\varphi}}$, as in the finite-temperature equilibrium case, consistent with what was obtained in Ref. [6] for critical quenches. This allows us to extract a time-scale $\tau_{Q}^{\varphi}$ characterizing the dynamics after the quench. This phase-coherence time depends in principle on the initial state $\left|\psi\left(\Gamma_{0}\right)\right\rangle$ and the final Hamiltonian $\mathcal{H}(\Gamma)$. However, and this is the main result of this Letter, all the information needed to characterize $\tau_{Q}^{\varphi}$ is encoded in two variables only: the final gap $\Delta(\Gamma)$, and an effective temperature $T_{\text {eff }}$. The latter is obtained by comparing the energy associated to the initial state with respect to the Hamiltonian after the quench to the average energy of a fictitious thermal state at temperature $T_{\text {eff }}$ in an effective canonical ensemble:

$$
E\left(\Gamma_{0}\right) \equiv\left\langle\psi\left(\Gamma_{0}\right)|\mathcal{H}(\Gamma)| \psi\left(\Gamma_{0}\right)\right\rangle=\langle\mathcal{H}(\Gamma)\rangle_{T_{\text {eff }}} .
$$

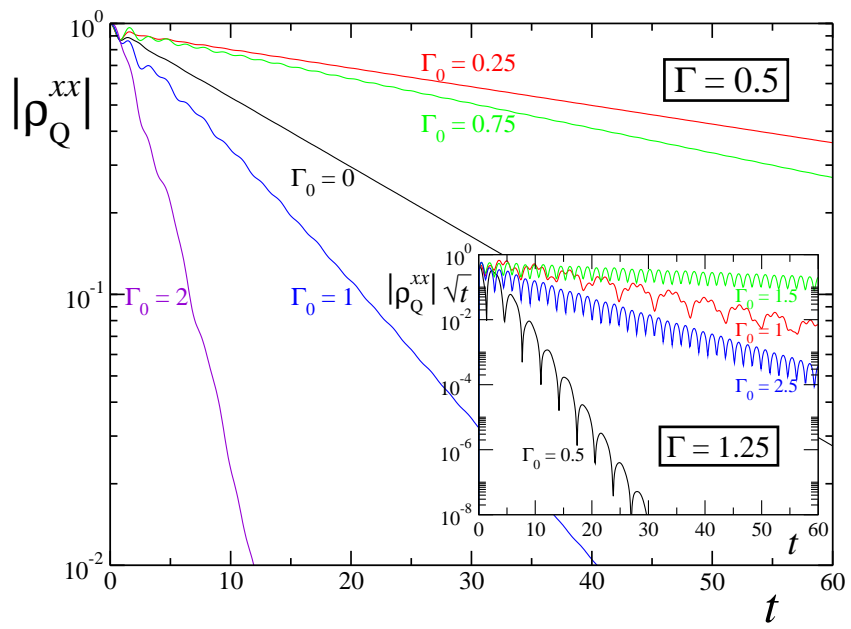

FIG. 1: (color online). Time dependence of $\left|\rho_{Q}^{x x}(t)\right|$ for a quench to a final ferromagnet $\Gamma=0.5$. Different curves, obtained numerically for a finite chain of $L=600$ sites, correspond to different initial $\Gamma_{0}$ 's. Inset: $\left|\rho_{Q}^{x x}(t)\right| \sqrt{t}$ for $\Gamma=1.25$.

Most importantly, we find that $\tau_{Q}^{\varphi}=\tau_{T=T_{\text {eff }}}^{\varphi}$, both for quenches at criticality and away from it.

To calculate $\rho_{Q}^{x x}(t)$ we exploit the complete integrability of the Ising chain [23, 24]. Here we sketch the essential steps [25]: first, one represents spins in terms of JordanWigner fermions $c_{l} \equiv \sigma_{l}^{-} \exp \left(i \pi \sum_{j=1}^{l-1} \sigma_{j}^{+} \sigma_{j}^{-}\right)$. Since the ground state has always an even number of fermions, one can focus on the even $c$-fermionic Hilbert space sector. Switching to momentum representation, the Hamiltonian is diagonalized with a Bogoliubov rotation: $\mathcal{H}(\Gamma)=$ $\sum_{k>0} \epsilon_{k}^{\Gamma}\left(\gamma_{k}^{\dagger} \gamma_{k}+\gamma_{-k}^{\dagger} \gamma_{-k}-1\right)$, where $\gamma_{k}$ are fermionic quasi-particle operators, $\epsilon_{k}^{\Gamma}=2 \sqrt{\Gamma^{2}-2 \Gamma \cos k+1}$ is their dispersion, and $k= \pm \frac{\pi(2 n+1)}{L}$ with $n=0, \ldots, \frac{L}{2}-1$. The second step consists in describing the dynamics after a quench. This can be easily done in the Heisenberg picture [26], by solving the closed set of equations of motion for the $c$-fermions in momentum space, with the initial conditions associated to the quench. Finally, $\rho_{Q}^{x x}(t)$ is computed using a trick developed in Ref. [25]. The operator $\sigma_{j}^{x}(t) \sigma_{j}^{x}(0)$ connects states with different $c$-fermion parity, and it cannot be simply evaluated using Jordan-Wigner fermions in the even Hamiltonian sector. This problem can be circumvented by considering a four-spin correlation function on a chain of length $L$, $C^{x}(t ; L)=\left\langle\sigma_{1}^{x}(t) \sigma_{1}^{x}(0) \sigma_{\frac{L}{2}+1}^{x}(t) \sigma_{\frac{L}{2}+1}^{x}(0)\right\rangle$. This correlator conserves the c-fermion parity, and can be written as the square root of a Pfaffian 25], using the techniques of Ref. [23]. One finally recovers $\rho_{Q}^{x x}(t)$ using the cluster property $\left[\rho_{Q}^{x x}(t)\right]^{2}=\lim _{L \rightarrow \infty} C^{x}(t ; L)$, by taking the square root of $C^{x}(t)$ in the limit of large number of spins.

As anticipated above, the zero-temperature quench autocorrelation $\rho_{Q}^{x x}(t)$ always relaxes exponentially to zero (see Fig. (1), irrespective of the initial state $\left|\psi\left(\Gamma_{0}\right)\right\rangle$ and of 


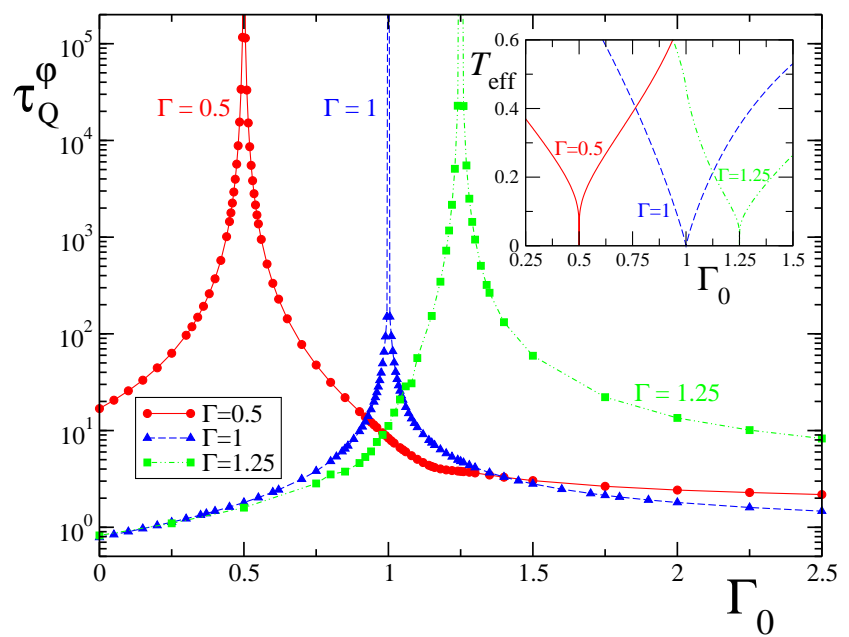

FIG. 2: (color online). Phase coherence time $\tau_{Q}^{\varphi}$ as a function of the initial transverse field $\Gamma_{0}$. The different curves refer to a ferromagnetic ( $\Gamma=0.5$, red circles), a critical $(\Gamma=1$, blue triangles) and a paramagnetic $(\Gamma=1.25$, green squares) quench dynamics. Inset: effective temperature $T_{\text {eff }}$ vs. $\Gamma_{0}$, as extracted from Eq. (3), for the same values of $\Gamma$.

the final transverse field $\Gamma \neq \Gamma_{0}: \rho_{Q}^{x x}(t) \sim e^{-t / \tau_{Q}^{\varphi}}$. This is in sharp contrast with the zero-temperature equilibrium autocorrelation $\rho_{T=0}^{x x}(t)$, which decays as $M_{x}^{2}+C / t$ for $\Gamma<1, M_{x}=\left(1-\Gamma^{2}\right)^{1 / 8}$ being the spontaneous magnetization [25]. Quenching to the paramagnetic side, the exponential drop is superimposed to an oscillatory power-law decay. This is once again reminiscent of finite-temperature equilibrium case, where $\rho_{T}^{x x}(t) \sim$ $K(t) e^{-t / \tau_{T}^{\varphi}}, K(t)$ being the quantum zero-temperature correlator [21], which oscillates and decays as $t^{-1 / 2}$. Indeed, for a quench to $\Gamma>1$, rescaling $\rho_{Q}^{x x}(t)$ with the zero-temperature factor $t^{-1 / 2}$, we recover exponential relaxation (inset of Fig. 1).

We now analyze the coherence time as a function of different initial and final conditions. In Fig. 2, $\tau_{Q}^{\varphi}$ is plotted as a function of initial $\Gamma_{0}$, for several final $\Gamma$ 's. A dramatic increase of $\tau_{Q}^{\varphi}$ as $\Gamma_{0} \rightarrow \Gamma$ is observed: the less the system goes out-of-equilibrium, the slower is the relaxation. If $\Gamma_{0}=\Gamma$, the exponential decay turns into a power-law, as in the zero-temperature equilibrium case, and $\tau_{Q}^{\varphi} \rightarrow \infty$. The analogy with the equilibrium finite-temperature behavior, where the lower is the temperature $T$ the longer is $\tau_{T}^{\varphi}$, is evident. It is therefore tempting to relate the two cases, by introducing an effective temperature $T_{\text {eff }}$ for the out-of-equilibrium system. We define $T_{\text {eff }}$ by comparing the energy of the initial state $\left|\psi\left(\Gamma_{0}\right)\right\rangle$ with that of a fictitious thermal state as in Eq. (3), with a thermal energy $\langle\mathcal{H}(\Gamma)\rangle_{T_{\text {eff }}}=\sum_{k>0} \epsilon_{k}^{\Gamma}\left(n_{k}\left(T_{\text {eff }}\right)+n_{-k}\left(T_{\text {eff }}\right)-1\right)$ determined by an effective canonical ensemble Fermi distribution function $n_{k}\left(T_{\text {eff }}\right)=\left(1+e^{\epsilon_{k}^{\Gamma} / T_{\text {eff }}}\right)^{-1}$ of the quasiparticles $\gamma_{k}$. A plot of $T_{\text {eff }}$ as a function of $\Gamma_{0}$, for different values of $\Gamma$, is shown in the inset of Fig. 2. Notice that,

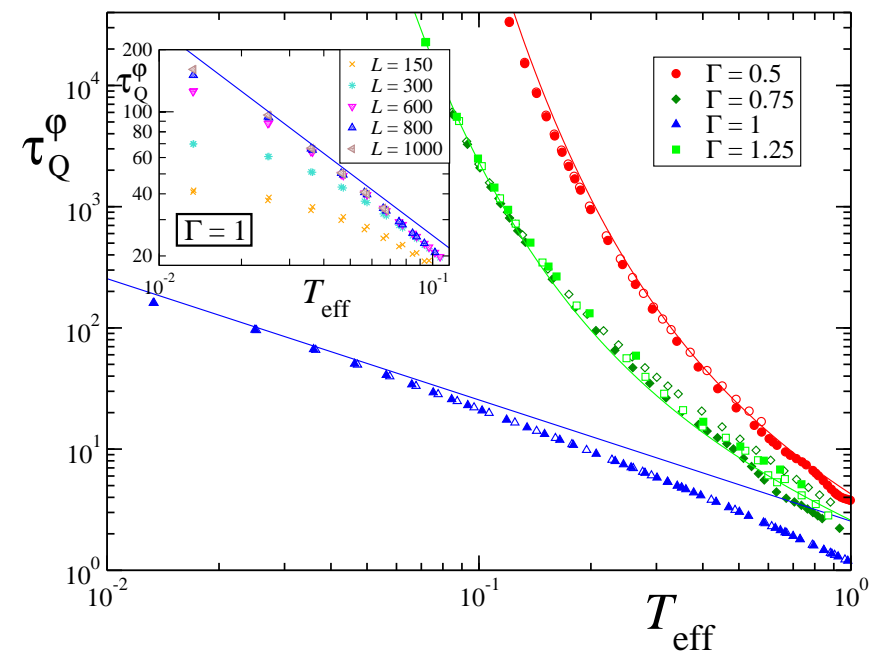

FIG. 3: (color online). Phase coherence time $\tau_{Q}^{\varphi}$ as a function of $T_{\text {eff }}$. Different symbols are for various values of $\Gamma$. Empty symbols correspond to an initial field $\Gamma_{0}<\Gamma$, while filled ones are for $\Gamma_{0}>\Gamma$. Straight lines denote the finite-temperature equilibrium values of $\tau_{T=T_{\text {eff }}}^{\varphi}$. The inset shows the finite-size scaling of $\tau_{Q}^{\varphi}$ at criticality. Data in the main panel are for $L=600$ (except for $\Gamma=1$ and $T_{\text {eff }}<0.07$, where $L=1000$ ).

for each $\Gamma$, there are two values of $\Gamma_{0}$ for which $T_{\text {eff }}$ is the same, one for $\Gamma_{0}<\Gamma$ and one for $\Gamma_{0}>\Gamma$.

The effective temperature $T_{\text {eff }}$, together with the quasiparticle gap $\Delta$ at the final $\Gamma$, univocally determines the phase coherence time $\tau_{Q}^{\varphi}$. Numerical evidence is shown in Fig. 3 points with equal $\Delta(\Gamma)$ have the same $\tau_{Q}^{\varphi}$ if effective temperatures are the same, even if $\Gamma_{0}$ and $\Gamma$ are different. Since the system is closed, it would be tempting to substitute $T_{\text {eff }}$ with just the initial energy $E\left(\Gamma_{0}\right)$. This is not always possible. Indeed, two quenches having different initial energy $E\left(\Gamma_{0}\right)$, but equal final gap $\Delta(\Gamma)$ and equal $T_{\text {eff }}$, will exhibit the same $\tau_{Q}^{\varphi}$ (see data for $\Gamma=1.25$ and 0.75 in Fig. 31). For example, the two quenches $1.21 \rightarrow 1.25$ and $0.715 \rightarrow 0.75$ have equal $T_{\text {eff }} \simeq 0.113$ within $0.7 \%$, (corresponding to $\tau_{Q}^{\varphi} \simeq 1170$ within $0.3 \%$ ), although their energies differ by $25 \%$. However, if the final $\Gamma$ is fixed, the canonical and microcanonical effective ensembles are equivalent, i.e., $E\left(\Gamma_{0}\right)$ and $T_{\text {eff }}$ can be interchanged. We also notice that, with a good accuracy, $\tau_{Q}^{\varphi}$ is still given by the equilibrium expressions at temperature $T_{\text {eff }}$, i.e., $\tau_{Q}^{\varphi} \simeq \tau_{T=T_{\text {eff }}}^{\varphi}$ out of criticality. In the critical case, a tendency to follow the equilibrium expressions is observed only at low temperatures, using a finite-size scaling (see inset of Fig. 31). This is necessary because, while the comparison is expected to work better at low $T_{\text {eff }}$, the consequent importance of the long wavelength modes makes finite-size effects more pronounced.

The time dependence of correlators is influenced by quasiparticle propagation, as exemplarily shown in a series of studies [4, 5, 6] focusing on the asymp- 


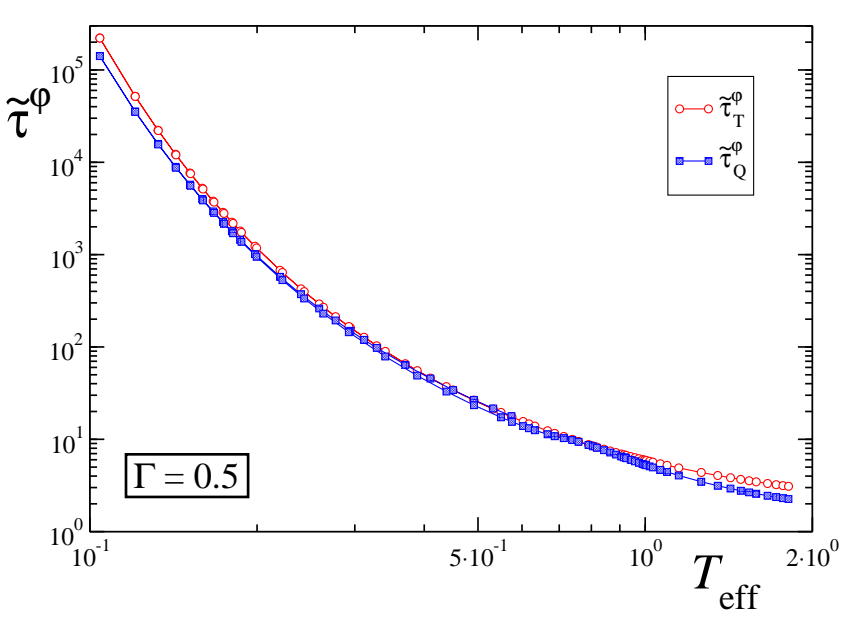

FIG. 4: (color online). Phase coherence time at $\Gamma=0.5$ computed according to the quasiparticle distribution $f_{k}\left(\tilde{\tau}_{Q}^{\varphi}\right.$, blue squares) and to the effective quasiparticle distribution $n_{k}\left(T_{\text {eff }}\right)\left(\tilde{\tau}_{T}^{\varphi}\right.$, red circles $)$.

totics of equal time correlators at different space-points, $\rho^{x x}(r, t)=\left\langle\sigma_{i+r}^{x}(t) \sigma_{i}^{x}(t)\right\rangle$. A similar picture, elucidating some of the results so far obtained and applicable in the off-critical regimes $\left(T_{\text {eff }} \ll \Delta\right)$, can be formulated in the spirit of Ref. 21]. When the transverse field is quenched, the initial condition for the time evolution consists of a state with a finite density of quasiparticles (relative to $\mathcal{H}(\Gamma)$ ), characterized by a dispersion $\epsilon_{k}^{\Gamma}$. For $\Gamma<1$, these can be seen as kinks propagating with momentum $k$ and velocity $v_{k}=\partial \epsilon_{k}^{\Gamma} / \partial k$. The correlator $\rho_{Q}^{x x}(t)$ is determined by the number of kinks passing through a single site in the interval $[0, t]$. A combinatorial analysis [21], together with an average over momenta, leads to $\rho_{Q}^{x x}(t) \simeq \exp \left[-t / \tilde{\tau}_{Q}^{\varphi}\right]$, with $\left(\tilde{\tau}_{Q}^{\varphi}\right)^{-1}=\frac{2}{L} \sum_{k}\left|v_{k}\right| f_{k}$, where $f_{k}=\left\langle\psi_{0}\left|\gamma_{k}^{\dagger} \gamma_{k}\right| \psi_{0}\right\rangle$ is the occupation of quasiparticle modes. Analogous arguments can be presented for $\Gamma>1$, giving $\rho_{Q}^{x x}(t) \simeq K(t) \exp \left(-t / \tilde{\tau}_{Q}^{\varphi}\right)$, where $K(t) \approx t^{-1 / 2}$ is the equilibrium zero-temperature correlator. Note that here integrability is not necessary: the same reasoning applies to the low-energy kink of non-integrable $\Phi^{4}$ theory that belongs to the same Ising universality class. While this picture explains the exponential decay towards zero, it is also important to notice the following: although the quasiparticle distribution function $f_{k}$ determined by the initial state and the effective thermal quasiparticle distribution function $n_{k}\left(T_{\text {eff }}\right)$ are typically very different, phase coherence times $\tilde{\tau}_{Q}^{\varphi}$ computed from $f_{k}$ or according to $\left(\tilde{\tau}_{T}^{\varphi}\right)^{-1}=\frac{2}{L} \sum_{k}\left|v_{k}\right| n_{k}\left(T_{\text {eff }}\right)$ are very close (see Fig. (4). In other words, we explicitly checked that, fixing an effective temperature through Eq. (3) or using $\tilde{\tau}_{Q}^{\varphi}=\tilde{\tau}_{T}^{\varphi}$, leads to qualitatively and quantitatively similar (to a few percent accuracy) results, for $\Delta \gg T_{\text {eff }} ;$ moreover, by imposing $\tilde{\tau}_{Q}^{\varphi}=\tilde{\tau}_{T}^{\varphi}$, we find $\tilde{T}_{\text {eff }} \sim 2 \Delta / \ln \left[\Delta /\left(\Gamma-\Gamma_{0}\right)^{2}\right]$ far from criticality and at low temperatures, in agreement with the cusp singularity of Fig. 2 .

In conclusion, we studied the phase coherence time $\tau_{Q}^{\varphi}$ after an abrupt quench of the transverse field in a Quantum Ising chain. We have shown that, irrespective of the integrability of the model, $\tau_{Q}^{\varphi}$ depends only on the quasiparticle gap $\Delta$ and the quasiparticle effective temperature $T_{\text {eff }}$, and provided numerical evidence of the fact that the dependence of $\tau_{Q}^{\varphi}$ on $T_{\text {eff }}$ is close to the one obtained at equilibrium as a function of the equilibrium temperature. The realization of the dynamics of artificial quantum spin chains using bosonic atoms in optical lattices 27] represents a concrete possibility to check our theoretical scenario with the available experimental tools.

We thank N. Andrei, E. Altman, R. Fazio, V. Oganesyan, V. Kravtsov, and A. Polkovnikov for discussions. G.M. acknowledges hospitality at Galileo Galilei Institute where this work was completed, and grants INSTANS (from ESF) and 2007JHLPEZ (from MIUR).

[1] I. Bloch et al., Rev. Mod. Phys. 80, 885 (2008).

[2] M. Greiner et al., Nature 415, 39 (2002); L. E. Sadler et al., Nature 443, 312 (2006).

[3] T. Kinoshita et al., Nature 440, 900 (2006).

[4] F. Iglói and H. Rieger, Phys. Rev. Lett. 85, 3233 (2000).

[5] K. Sengupta et al., Phys. Rev. A 69, 053616 (2004).

[6] P. Calabrese and J. Cardy, Phys. Rev. Lett. 96, 136801 (2006); J. Stat. Mech.: Theory Exp. (2007) P06008.

[7] M. Rigol et al., Phys. Rev. Lett. 98, 050405 (2007); M. Rigol et al., Phys. Rev. A 74, 053616 (2006).

[8] M. Rigol et al., Nature 452, 854 (2008).

[9] C. Kollath et al., Phys. Rev. Lett. 98, 180601 (2007).

[10] S. R. Manmana et al., Phys. Rev. Lett. 98, 210405 (2007).

[11] M. Cramer et al., Phys. Rev. Lett. 100, 030602 (2008).

[12] T. Barthel and U. Schollwöck, Phys. Rev. Lett. 100, 100601 (2008).

[13] M. Eckstein and M. Kollar, Phys. Rev. Lett. 100, 120404 (2008); M. Kollar and M. Eckstein, Phys. Rev. A 78, 013626 (2008).

[14] M. A. Cazalilla, Phys. Rev. Lett. 97, 156403 (2006).

[15] D. M. Gangardt and M. Pustilnik, Phys. Rev. A 77, 041604(R) (2008).

[16] S. Sachdev, Quantum Phase Transitions, (Cambridge University Press, Cambridge, 2000).

[17] J. B. Zuber and C. Itzykson, Phys. Rev. D 15, 2875 (1977).

[18] V. P. Yurov and Al. B. Zamolodchikov, Int. J. Mod. Phys. A 6, 3419 (1991).

[19] G. Mussardo, Nucl. Phys. B 779, 101 (2007).

[20] P. Deift and X. Zhou, in Singular limits of dispersive waves (Lyon, 1991), 183, NATO Adv. Sci. Inst. Ser. B Phys. 320, Plenum, New York, 1994.

[21] S. Sachdev and A. P. Young, Phys. Rev. Lett. 78, 2220 (1997).

[22] Given the bandwidth of the excitation $W=2 J(|1+\Gamma|-$ $|1-\Gamma|)$ we assume $T, T_{\text {eff }} \ll W$.

[23] E. Lieb et al., Ann. Phys. 16, 407 (1961). 
[24] P. Pfeuty, Ann. Phys. 57, 79 (1970).

[25] B. M. McCoy et al., Phys. Rev. A 4, 2331 (1971).

[26] E. Barouch et al., Phys. Rev. A 2, 1075 (1970).
[27] L. M. Duan et al., Phys. Rev. Lett. 91, 090402 (2003). 